\title{
INFORMACION
}

\section{Modificaciones producidas en las proteínas alimentarias por su interacción con lípidos peroxidados. I. Química radicalaria de los ácidos grasos poliinsaturados.}

\author{
Por F. J. Hidalgo, R. Zamora y M. Alaiz
}

Instituto de la Grasa y sus Derivados. C.S.I.C.

Avda. Padre García Tejero, 4. 41012 - Sevilla

RESUMEN

Modificaciones producidas en las proteinas alimentarias por su interacción con lipidos peroxidados. I. Química radicalaria de los ácidos grasos poliinsaturados.

En esta parte se hace una revisión de los mecanismos de oxidación lipídica haciendo especial hincapié en cómo los diversos compuestos formados pueden reaccionar con las proteínas.

PALABRAS-CLAVE: Acido graso peroxidado - Alimento Información (artículo) - Producto secundario - Acido graso peroxidado.

\section{SUMMARY}

Modifications produced in food proteins following interactions with oxidizing lipids. I. Radical chemistry of polyunsaturated fatty acids.

This part summarizes the mechanisms of lipid oxidation. The compounds that react with proteins are specially discussed

KEY-WORDS: Food - Information (paper) - Oxidized fatty acid - Protein - Secondary product.

\section{INTRODUCCION}

La oxidación lipídica es una de las mayores causas del deterioro de los alimentos. Es, por una parte, de gran importancia económica porque causa, en aceites comestibles y en alimentos ricos en grasas, el desarrollo de olores y aromas desagradables que son los causantes de la rancidez y hacen a los alimentos que los contienen inaceptables o, en el mejor de los casos, reduce en gran medida su vida comercial. Al mismo tiempo estas reacciones oxidativas pueden decrecer la calidad nutricional de los alimentos y ciertos productos de oxidación son potencialmente tóxicos. Por el contrario, y en determinados casos, un cierto grado de oxidación lipídica es, a veces, deseable como ocurre en la producción de ciertos quesos o en los aromas de fritura (1).

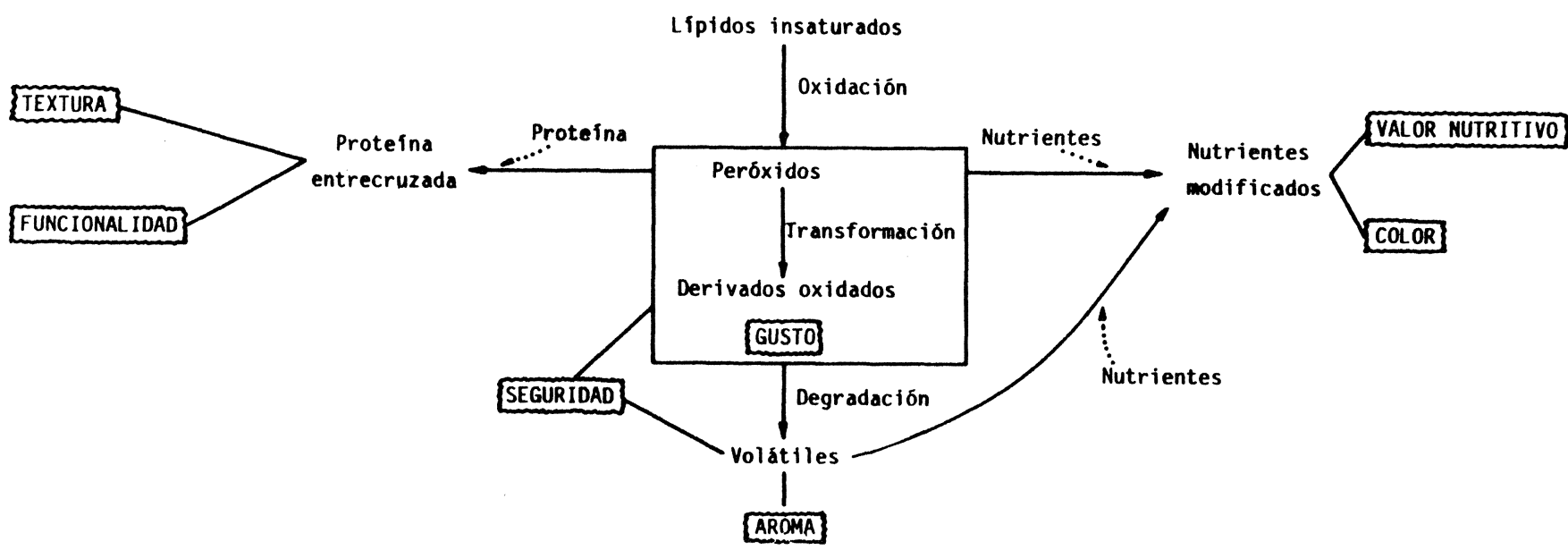

Figura 1

Modificaciones producidas en los alimentos como consecuencia de la oxidación lipídica y su interacción con proteínas y otros componentes (Adaptado de referencia 2). 
Por todas estas razones es muy extensa la investigación que ha sido llevada a cabo no sólo para identificar los productos de oxidación lipídica sino también las condiciones que influyen en su producción así como el estudio de los mecanismos que están envueltos.

No obstante, tanto en los alimentos como en otros sistemas biológicos los lípidos no se encuentran como sustratos puros en un matraz sino que se hayan rodeados de materiales no lipídicos tales como proteínas, carbohidratos, agua, enzimas, sales, vitaminas y pro- y antioxidantes con los que pueden reaccionar (2). Todo esto hace que la reacción de oxidación lipídica se complique y produzca una serie de efectos en las propiedades de los alimentos que se han tratado de esquematizar en la Figura 1.

El objetivo de esta revisión es una aproximación al estado actual del conocimiento de cómo se producen estas reacciones a nivel molecular, centrándonos especialmente en la degradación de proteínas por lípidos peroxidados que es un tema que ha venido preocupando en los últimos años a nuestro grupo (3-9) por la incidencia que el mismo tiene en diversos alimentos como pueden ser pescados, cereales (10), semillas oleaginosas (11), etc.

La primera parte de este estudio estará dedicada a la discusión de los mecanismos de oxidación lipídica con objeto de identificar los diversos productos formados en la oxidación de los lípidos que son los que a la postre van a reaccionar con los grupos activos libres de las proteínas.

\section{2. ¿POR QUE SE OXIDAN LOS LIPIDOS?}

Las oxidaciones espontáneas son en general una consecuencia de la naturaleza especial de la molécula de oxígeno (12). En su estado normal, esta molécula tiene dos electrones desapareados que se encuentran en estado triplete. A causa de esta rara característica, el oxígeno es un eficiente captor de radicales.

No obstante, un ácido graso insaturado no se oxidará en presencia de oxígeno a menos que le sea arrancado un protón para formar un radical. Esta abstracción, que puede ser producida enzimáticamente o no, inicia una cadena radicalaria (que se ha representado en la Figura 2) y cuyas consecuencias rebasan el campo de la química y bioquímica de alimentos para pasar a jugar un importante papel en organismos vivos en un campo que hoy en día se haya en plena efervescencia.

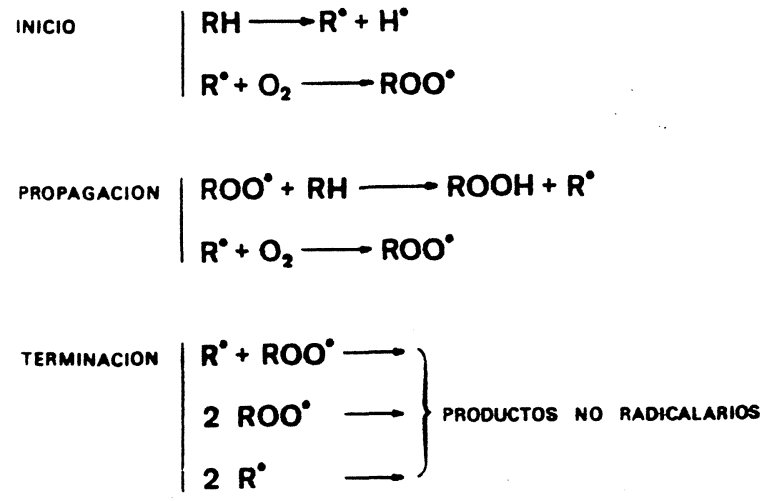

Figura 2.

Mecanismo radicalario de oxidación de un ácido graso.

Puesto que un enlace $\mathrm{CH}$ de un carbono alílico tiene una energía de disociación relativamente débil, éste es el protón que sale en la molécula de ácido graso. El correspondiente radical alílico formado se combina con oxígeno para producir un radical peroxilo, el cual propaga la cadena por nuevas abstracciones de protones en moléculas de ácidos grasos. Así se forma el hidroperóxido lipídico que es susceptible de una disociación homolítica por pérdida de un protón o de un radical hidroxilo. En el primer caso la reacción es habitualmente reversible mientras que no ocurre lo mismo en el segundo caso. En cualquiera de los dos casos, al igual que ocurre con los diversos productos formados a partir del radical peroxilo, se produce la transformación de estos radicales bien en compuestos más estables o bien sufren una rotura para dar lugar a productos secundarios de oxidación a la vez que se generan nuevos radicales.

El progreso de la autooxidación hipotética de un lípido es por tanto, la sucesión de una serie de reacciones que, aunque consecutivas, son al mismo tiempo simultáneas. La fase de iniciación es seguida por una rápida acumulación de radicales que promueven a la vez la formación y la destrucción de los hidroperóxidos. Finalmente, la combinación de radicales conduce a la formación de productos secundarios no radicalarios. Como discutiremos en la segunda parte de este estudio, ambos, productos secundarios y reacciones radicalarias, están envueltos en el deterioro de los alimentos producido por su reacción con las proteínas. 


\section{MECANISMOS DE LA OXIDACION LIPIDICA}

Existen varios caminos de reacción que conducen a los diversos productos secundarios de oxidación lipídica, de los cuales se van a describir los principales. El objetivo es ver la diversidad de productos que son producidos en estas reacciones. Por eso mismo, la discusión será de alguna manera general y se hará especial hincapié en los productos que reaccionan con las proteínas. Recientemente han aparecido buenas revisiones bibliográficas sobre este tema que dan una visión más general de la que se pretende dar aquí (13-16).

\subsection{Reacciones de los radicales peroxilos}

\subsubsection{Abstracción de átomos de hidrógeno}

Una importante reacción producida por los radicales peroxilos es la abstracción de átomos de hidrógeno que ayuda a propagar la cadena. Esta abstracción puede tener lugar sobre el hidrógeno alílico de un ácido graso, para formar un radical alílico, o sobre cualquier otro hidrógeno débilmente enlazado que se encuentre en las proximidades dando lugar a un nuevo radical. Puesto que $\mathrm{XH}$ es frecuentemente un compuesto no lipídico que se oxida en presencia de lípidos peroxidantes, este proceso es, algunas veces, llamado cooxidación. Esta reacción tiene mucho interés cuando el compuesto $\mathrm{XH}$ es una proteína ya que diferentes aminoácidos, como por ejemplo lisina, arginina, histidina, triptófano y cisteína, son buenos receptores de este radical libre dando lugar a diversas reacciones que serán comentadas con posterioridad. El resultado final de esta propagación en cadena es la destrucción de diversos aminoácidos, lo que a la postre repercute en el valor nutritivo; y el entrecruzamiento de las cadenas polipeptídicas, que da lugar a cambios en la textura.

\subsubsection{Escisiones en B}

Las reacciones de escisión en $B$ ocurren como el paso inverso a la propagación y es difícil saber qué impacto puede tener en alimentos.

\subsubsection{Reordenamiento intramolecular}

Estas reacciones si pueden tener más importancia en sistemas alimenticios. El reordenamiento ocurre si hay un doble enlace en $B$ al carbono

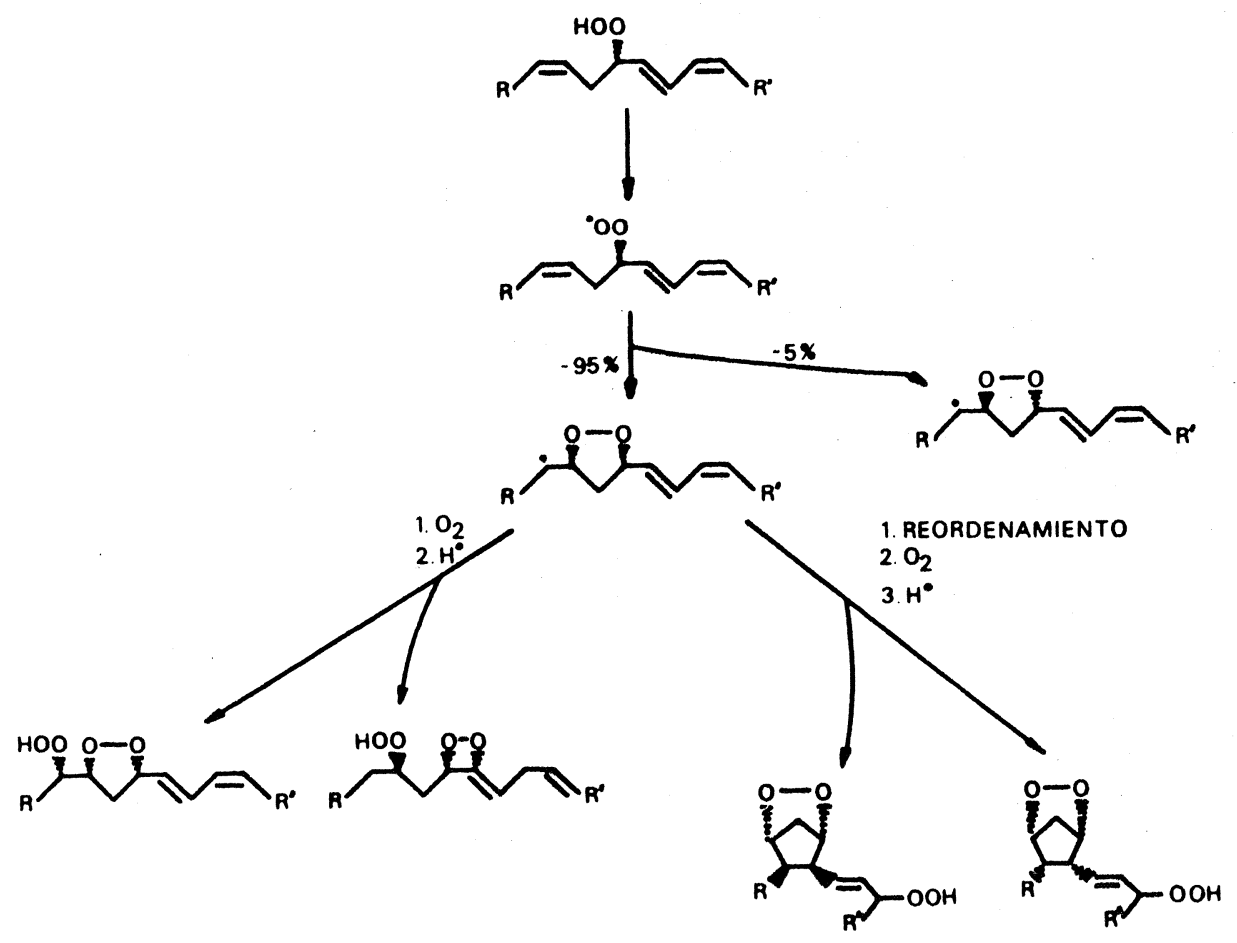

Figura 3

Esquema propuesto para la formación de peróxidos ciclicos y cicloendoperóxidos. 
portador del grupo peroxilo (Fig. 3). Esto conduce a la formación de peróxidos cíclicos (17) (18) y endoperóxidos del tipo de las prostaglandinas (19). Estos compuestos se cree que son importantes en la génesis del malondialdehído (20), en la propagación radicalaria y en la formación de otros productos secundarios. La importancia de la formación del malondialdehído al provocar fluorescencia y entrecruzamiento en proteínas será discutida con posterioridad.

Como se aprecia en la Fig. 3, la estructura necesaria para el reordenamiento requiere hidroperóxidos interiores de ácidos grasos poliinsaturados con 3 o más dobles enlaces. (Aunque no va a ser tratado aquí, el reordenamiento también puede tener lugar con hidroperóxidos no conjugados obtenidos por oxidación con oxígeno singlete de ácidos grasos poliinsaturados con dos o más dobles enlaces) (18). Por tanto, y a pesar de ciertas propuestas que aparecen en la literatura, el radical peroxilo del hidroperóxido conjugado del ácido linoleico no se reordena intramolecularmente con alguno de los dobles enlaces: se puede decir que de alguna manera el doble enlace trans $\alpha, \beta$ separa el radical peroxilo del doble enlace $\gamma, \delta$. Por esta razón el malóndialdehído no es generado a partir del hidroperóxido conjugado del ácido linoleico (21).

Si el ácido graso poliinsaturado tiene cuatro o más dobles enlaces entonces puede sufrir una serie de ciclaciones sucesivas dando lugar a peróxidos ciclicos en diversas partes de la molécula (22) (23).

\subsubsection{Combinación para formar un tetraóxido}

Los radicales peroxilos también pueden combinarse para formar un tetraóxido inestable (ROOOOR) (24) que se descompone posteriormente a través de un estado de transición cíclico para dar lugar a una cetona (RCOR), un alcohol $(\mathrm{RCH}(\mathrm{OH}) \mathrm{R})$ y una molécula de oxígeno singlete.

Esta combinación radicalaria ha sido sugerida en compuestos modelo por estudios con isótopos (25) y por resonancia de espín electrónico a $-80^{\circ} \mathrm{C}$ (26). Es asimismo aplicable a radicales peroxilos derivados de los hidroperóxidos lipídicos ya que el enlace $\mathrm{C}-\mathrm{H}$ necesario para completar el estado de transición está presente. Para conservar el spín, la rotura del tetraóxido debe producir directa o indirectamente oxígeno singlete $(27,28)$. La formación de ${ }^{1} \mathrm{O}_{2}$ ha sido observada por quimiluminiscencia coincidente con la producción de radicales peroxilos a partir del hidroperóxido del ácido linoleico aunque con un rendimiento menor de lo esperado (29) (30).

\subsubsection{Adición intermolecular a un doble en- lace}

La adición intermolecular del radical peroxilo a un doble enlace, es posible aunque no se haya documentado en detalle para lípidos. Sin embargo, se presume que la polimerización de poliinsaturados puede tener lugar de esta manera (31).

Esta reacción puede dar lugar asímismo a epóxidos por un mecanismo análogo al de la Figura 4 que ha sido propuesto para un hidroperóxido no conjugado del linoleato de metilo (18). Estos epóxidos de ácidos grasos conteniendo otros grupos oxigenados se aislan habitualmente después de la descomposición de los radicales peroxilos. No obstante, existen otros mecanismos alternativos para la formación de estos compuestos (algunos de los cuales serán comentados posteriormente) por lo que el origen del oxígeno de los epóxidos es difícil de deducir.
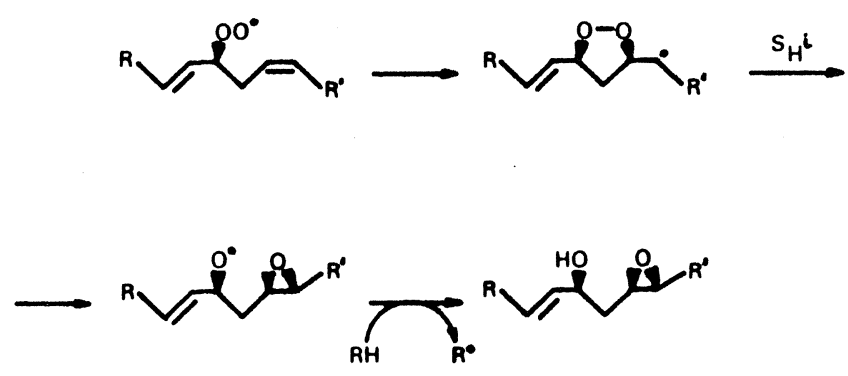

Figura 4

Mecanismo propuesto para la adición intramolecular de un radical peroxi a una olefina.

\subsection{Reacciones de los radicales alcoxilos}

Los radicales alcoxi son formados por rotura homolítica de los hidroperóxidos a través de diferentes mecanismos, principalmente de manera térmica o por reducción con metales de transición (32). Estos radicales son muy reactivos y se sabe que toman parte en diversos procesos competitivos radicalarios.

\subsubsection{Abstracción de átomos de hidrógeno}

Al igual que los radicales peroxilo, estos radicales pueden abstraer un átomo de hidrógeno para formar otros radicales en reacciones análogas a las descritas en 3.1.1. 


\subsubsection{Desproporción}

Un radical peroxilo puede abstraer un hidrógeno de otro radical para dar lugar a una cetona $y$ él pasar a alcohol. Ambos productos (cetonas y alcoholes) se encuentran entre los productos secundarios de oxidación lipídica y pueden reaccionar con las proteínas como se discutirá posteriormente.

\subsubsection{Escisión en $B$}

Análogamente a los radicales peroxilos, los alcoxi también pueden sufrir una escisión en $B$. Esta reacción genera aldehídos volátiles e hidrocarburos (33-35). Aunque los volátiles no exceden normalmente del $10-15 \%$ del lípido oxidado, la porción aldehídica de los volátiles ha recibido una extraordinaria atención en la bibliografía a causa de su contribución a los olores rancios. En la parte II de esta serie será discutida cómo su importancia no sólo estriba en esto sino que también son muy probablemente los responsables de la aparición de fluorescencia en proteínas, de parte de la pérdida de su valor nutritivo y de la formación de compuestos macromoleculares coloreados en las mismas.

\subsubsection{Formación de dímeros unidos por un} oxígeno de tipo etéreo

Aunque durante algún tiempo se ha supuesto que la formación de dímeros del tipo ROR procedía de la combinación de radicales alcoxi y alquilo, recientes investigaciones paracen apoyar un mecanismo de adición intermolecular de un radical alcoxi a uñ doble enlace (16).

\subsubsection{Adición intramolecular a olefinas}

Los radicales alcoxi son también capaces de adicionarse a olefinas por mecanismos intramole- culares para dar lugar a un radical epoxialílico (3638). Este radical ha sido atrapado y caracterizado por RSE (39). Puesto que el oxígeno es un excelente atrapador de radicales, el radical epo-

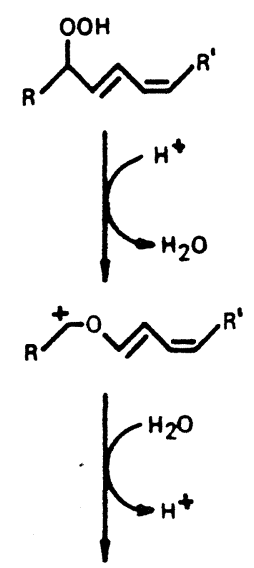<smiles>[2H]C(O)OC=CC=CP</smiles><smiles>[R]C/C=C/C(=O)/C=C/CC(=O)/C=C/[3H]</smiles>

Figura 5

Transposición de Hock/Criegee para un hidroperóxido lipídico en medio ácido.

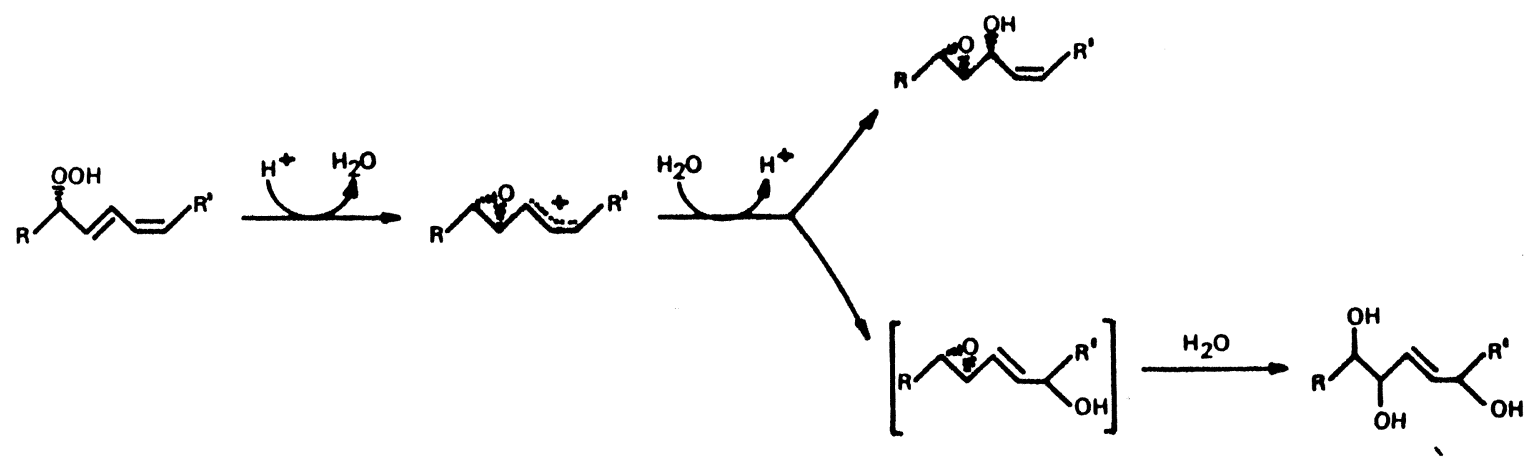

Figura 6

Mecanismo propuesto para la formación de epóxidos en medio ácido por reacciones heterolíticas. 
Tabla 1

Compuestos volatiles identificados en mezclas de acido linoleico y linoleato de metilo peroxidados calentados a temperaturas moderadas*

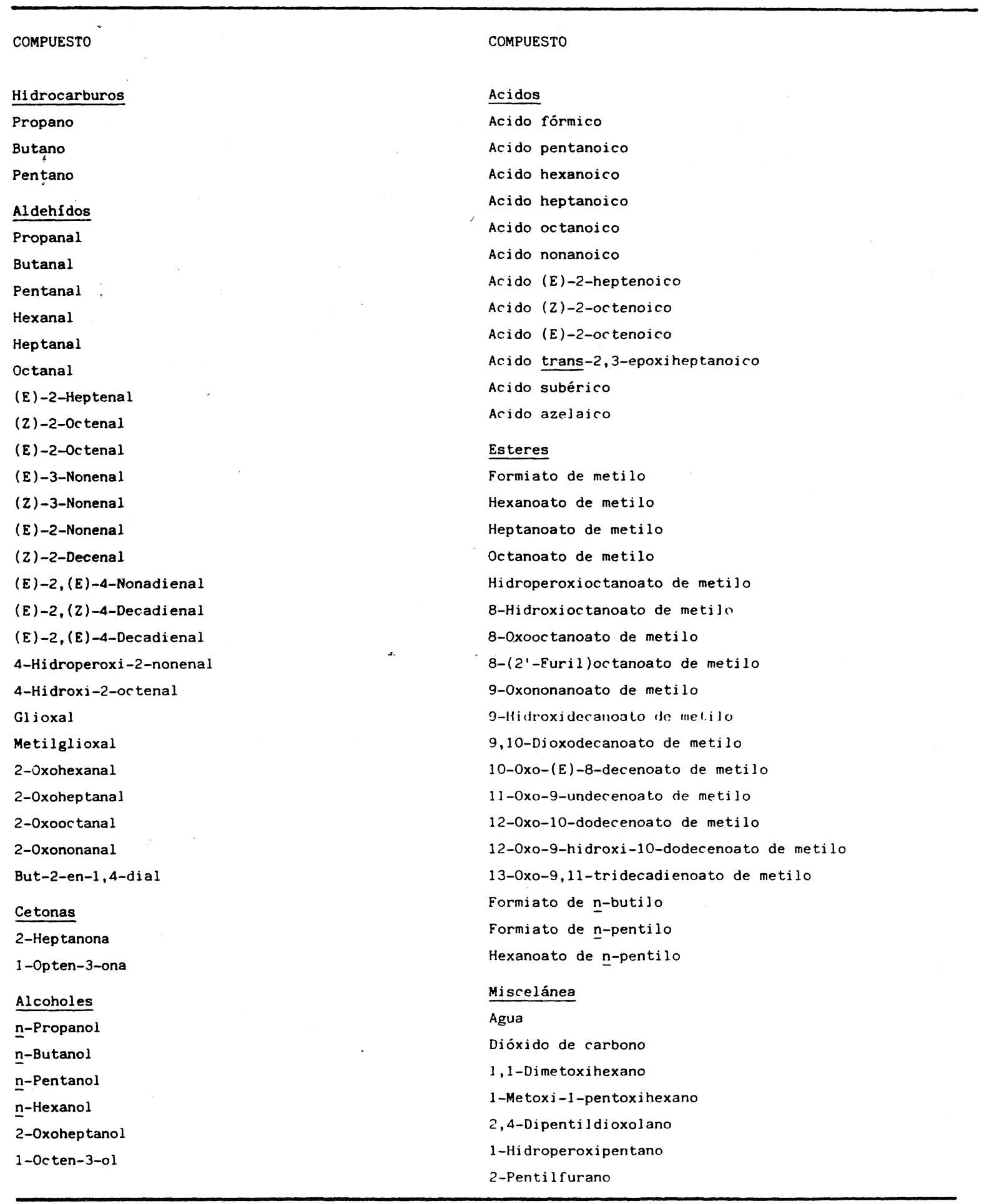

- Tomado de referencia 35. 
xialílico formado puede ser posteriormente oxidado para dar lugar a un epoxihidroperóxido. Este nuevo grupo hidroperóxido puede sufrir posterior homólisis y podría dar lugar de nuevo a todas las reacciones anteriormente descritas.

\subsection{Reacciones heterolíticas}

Aparte de las reacciones homolíticas que han sido descritas previamente, los hidroperóxidos son también susceptibles de sufrir reacciones heterolíticas dando lugar a productos que pueden ser similares a aquellos obtenidos por homólisis.

El anión hidroxi así como otros nucleófilos son capaces de transformar el grupo hidroperóxido en un grupo hidroxi (40).

En medio ácido los hidroperóxidos pueden sufrir un reordenamiento, la transposición de Hock/Criegee, dando lugar a un éter vinílico que se descompone a un aldehído y un oxoácido (Figura 5). Así, por ejemplo, el tratamiento ácido del éster metílico del 13-hidroperóxido del ácido linoleico dio hexanal y 12-oxo-cis-9-dodecenoato de metilo, en el que la olefina 9-cis esperada se isomerizó a 10trans en el medio ácido (41). Este reordenamiento puede evolucionar igualmente hacia la formación de epóxidos (Figura 6) (42).

Los hidroperóxidos pueden también epoxidar dobles enlaces de las olefinas por ataque heterolítico provocado por la nucleofilia de la olefina. Esta reacción es catalizada por metales de transición (43) (44).

\section{CONCLUSION}

La gran variedad de reacciones que pueden sufrir estos radicales hace que el número de compuestos obtenidos, aun partiendo de muestras de ácidos grasos puros, sea enorme. La Tabla I recoge los compuestos volátiles que han sido identificados en muestras de ácido linoleico o linoleato de metilo peroxidado. Las muestras fueron dejadas a temperatura ambiente o calentadas a un máximo de $40^{\circ} \mathrm{C}$. Entre los productos identificados se encuentran hidrocarburos, aldehídos saturados, monoinsaturados, diinsaturados, hidroxialdehídos, aldehídohidroperóxidos, cetoaldehídos, dialdehídos, cetonas, alcoholes, alcoholes insaturados, ácidos, ácidos insaturados, ésteres, hidroxiésteres, oxoésteres, oxohidroxiésteres, y hasta compuestos heterocíclicos. Y sólo se trata de los volátiles producidos por un ácido graso puro. En el momento en que pasamos a un alimento donde hay diver- sos ácidos grasos y que además están en contacto con material no lipídico, el número de productos se eleva exponencialmente hasta un cifra difícil de determinar. Los mecanismos de interacción entre estos productos de oxidación lipídica y los aminoácidos, péptidos y proteínas serán abordados en la parte II de este estudio.

\section{AGRADECIMIENTOS}

Al Prof. Eduardo Vioque por sus valiosas discusiones durante la realización de este trabajo.

\section{BIBLIOGRAFIA}

1. Nawar, W. W.- "Lipids" en "Food Chemistry", pp. 139-244.Fennema, O. R. (Ed.), Marcel Dekker, Inc., New York 1985.

2. Eriksson, C. E.- "Oxidation of lipids in food systems" en "Autoxidation of Unsaturated Lipids", pp. 207-231.- Chan, H. W. S. (Ed.), Academic Press, London 1987.

3. Zamora, R.; Millán, F.; Hidalgo, F. J.; Alaiz, M.; Maza, M. P. y Vioque, E.- "Aplicaciones de la fluorescencia al estudio de las interacciones lípido-proteína. Ensayos previos".Grasas y Aceites 37 (1986) 317-319.

4. Zamora, R.; Hidalgo, F. J.; Alaiz, M.; Millán, F.; Maza, M. P. y Vioque, E.- "Interacción entre el glutatión y diferentes oxoácidos grasos".- Grasas y Aceites 38 (1987) 318-322.

5. Zamora, R.; Alaiz, M.; Hidalgo, F. J.; Millán, F.; Maza, M. P. y Vioque, E.- "Empleo de lípidos oxidados hidrofílicos en los estudios de interacción lípido-proteínan.- Grasas y Aceites 38 (1987) 400-403.

6. Zamora, R.; Hidalgo, F. J.; Alaiz, M.; Millán, F.; Maza, M. P. y Vioque, E.- "Estudio de la interacción entre el ácido alfa-eleoesteárico y el glutatión".- Grasas y Aceites 39 (1988) 35-38.

7. Zamora, R.; Millán, F.; Hidalgo, F. J.; Alaiz, M.; Maza, M. P.; Olias, J. M. y Vioque, E.- "Interaction between the peptide glutathione and linoleic acid hydroperoxide ${ }^{n}$.- Nahrung 33 (1989) 283-288

8. Hidalgo, F. J. y Kinsella, J. E.- "Changes in B-lactoglobulin $B$ following interactions with linoleic acid 13-hydroperoxide".- J. Agric. Food Chem. 37 (1989) 860-866.

9. Hidalgo, F. J.; Zamora, R.; Alaiz, M.; Maza, M. P.; Millán, F. y Vioque, E.- "Non-enzymatic browning reactions of phospholipids".- Fett. Wissenchaft Technol. 92 (1990) 185188.

10. Harrison, B. N.; Pla, G. W.; Clark, G. A. y Fritz J. C."Selection of iron sources for cereal enrichment".- Cereal Chem. 53 (1976) 78-84.

11. St. Angelo, A. J.; Kuck, J. C. y Ory, R. L.- "Role of lipoxygenase and lipid oxidation in quality of oilseeds".- J. Agric. Food Chem. 27 (1979) 229-234.

12. Lowry, T. H. y Richardson, K. S.- Mechanism and theory in organic chemistry. 2nd edition.- pp. 714-716. Harper \& Row, Publishers, New York, 1981.

13. Frankel, E. N.- "Chemistry of free radical and singlet oxidation of lipids".- Prog. Lipid Res. 23 (1985) 197-221.

14. Porter, N. A.- "Mechanisms for the autoxidation of polyunsaturated lipids".- Acc. Chem. Res. 19 (1986) 262-268. 
15. Chan, H. W. S.- Autoxidation of unsaturated lipids.- Academic Press, London, 1987.

16. Gardner, H. W.- "Oxygen radical chemistry of polyunsaturated fatty acids".- Free Rad. Biol. Med.- 7 (1989) 65-86.

17. Chan, H. W. S.; Matthew, J. A. y Coxon, D. T.- "A hydroperoxyepidioxide from the autoxidation of a hydroperoxide of methyl linoleaten.- J. Chem. Soc. Chem. Commun. (1980) 235-236.

18. Mihelich, E. D.- "Structure and stereochemistry of novel endoperoxides isolated from the sensitized photooxidation of methyl linoleate. Implications for prostaglandin biosynthesis".- J. Am. Chem. Soc. 102 (1980) 7141-7143.

19. O'Connor, D. E.; Mihelich, E. D. y Coleman, M. C.- "Isolation and characterization of bicycloendoperoxides derived from methyl linoleate".- J. Am. Chem. Soc. 103 (1981) 223224.

20. Pryor, W. A. y Stanley, J. P.- "Suggested mechanism for the production of malonaldehyde during the autoxidation of polyunsaturated fatty acids. Nonenzymatic production of prostaglandin endoperoxides during autoxidation".- J. Org. Chem. 40 (1975) 3615-3617.

21. Dahle, L. K.; Hill, E. G. y Holman, R. T.- "The thiobarbituric acid reaction and the autoxidation of polyunsaturated fatty acid methyl esters".- Arch. Biochem. Biophys. 98 (1962) 253-261

22. Khan, J. A.; Porter, N. A.- "Serial cyclizations of an arachidonic acid hydroperoxide".- Angew. Chem. Int. Ed. Engl. 21 (1982) 217-218

23. Frankel, E. N.; Neff. W. E. y Weisleder, D.- "Formation of hydroperoxy bis-epidioxides in sensitized photooxidized methyl linoleate".- J. Chem. Soc. Chem. Commun. (1982) 599-600.

24. Russel, G. A.- "Deuterium-isotope effects in the autoxidation of aralkyl hydrocarbons. Mechanism of the interaction of peroxy radicals".- J. Am. Chem. Soc. 79 (1957) 3871 3877.

25. Bartlett, P. D. y Traylor, T. G.- "Oxygen-18 tracer studies of alkylperoxy radicals. I. The cumylperoxy radical and chain termination in the autoxidation of cumene".- J. Am. Chem. Soc. 85 (1963) 2407-2410.

26. Bartlett, P. D. y Guaraldi, G.- "Di-tert-butyl trioxide and ditert-butyl tetraoxiden.- J. Am. Chem. Soc. 89 (1967) 4799 4801.

27. Howard, J. A. y Ingold, K. U.- "The self-reaction of secbutyl peroxy radicals. Confirmation of the Russell mechanism".- J. Am. Chem. Soc. 90 (1968) 1056-1059.

28. Kellogg, R. E.- "Mechanism of chemiluminescence from peroxy radicals".- J. Am. Chem. Soc. 91 (1969) 5433-5436.

29. Nakano, M.; Takayama, K.; Shimizy, Y.; Tsuji, Y. y Inaba, H.- Spectroscopic evidence for the generation of singlet oxygen in self-reaction of sec-peroxy radicals".- J. Am. Chem. Soc. 98 (1976) 1974-1975.

30. Kanofsky, J. R.-"Singlet oxygen production from the reactions of alkyperoxy radicals. Evidence from $1268 \mathrm{~nm}$ chemiluminescencen.- J. Org. Chem. 51 (1986) 3386-3388.
31. Gardner, H. W- "Effects of lipids hydroperoxides on food components" en "Xenobiotics in Food and Feeds". pp. 6384.- Finley, J. W. y Schwass, D. E. (Eds.), A.C.S. Symp. Series 234. American Chemical Society, Washington D. C. 1983.

32. Aust, S. D.; Morehouse, L. A.; Thomas, C. E.-"Role of metals in oxygen radical reactions ${ }^{n}$.- J. Free Radicals Biol. Med. 1 (1985) 3-25.

33. Frankel, E. N.- "Volatile lipid oxidation products".- Prog. Lipid Res. 22 (1983) 1-33.

34. Min, D. B.; Smouse, T. H.- Flavor Chemistry of fats and oils.- American Oil Chemists' Society, Champaign (Illinois), 1985.

35. Grosch, W.- "Reactions of hydroperoxides-products of low molecular weight" en "Autoxidation of unsaturated lipids", pp. 95-139.- Chan, H. W.- S. (Ed.), Academic Press, London, 1987.

36. Gardner, H. W.; Eskins, K; Grams, G. y Inglett, G. E."Radical addition of linoleic hydroperoxides to $\alpha$ - tocopherol or the analogous hydroxychroman".- Lipids 7 (1972) 324 334 .

37. Gardner, H. W.; Kleiman, R. y Weisleder, D.- "Homolytic decomposition of linoleic acid hydroperoxide. Identification of fatty acid products".- Lipids 9 (1974) 696-706.

38. Hamberg, M.- "Decomposition of unsaturated fatty acid hydropexides by hemoglobin: Structures of major products of $13 \mathrm{~L}$-hydroperoxy-9,11-octadecadienoic acid". - Lipids 10 (1975) 87-92.

39. Schreiber, J.; Mason, R. P. y Eling, T. E.- "Carbon-centered free radical intermediates in the hematin- and ram seminal vesicle-catalyzed decomposition of fatty acid hydroperoxides".- Arch. Biochem. Biophys. 251 (1986) 17-24.

40. O'Brien, P. J.- "Intracellular mechanism for the decomposition of a lipid peroxide. I. Decomposition of a lipid peroxide by metal ions, heme compounds, and nucleophiles". Can. J. Biochem. 47 (1969) 485-499.

41. Gardner, H. W. y Plattner, R. D.- "Linoleate hydroperoxides are cleaved heterolytically into aldehydes by a Lewis acid in aprotic solvent".- Lipids 19 (1984) 294-299.

42. Gardner, H. W.; Nelson, E. C.; Tjarks, L. W. y Englands, R. E.- "Acid-catalyzed transformation of 13(S)-hydroperoxilinoleic acid into epoxyhydroxyoctadecenoic and trihydroxyoctadecenoic acids ${ }^{n}$.- Chem. Phys. Lipids. 35 (1984) $87-$ 101.

43. Mercier, J. y Agoh, B.- "Comportment d'hydroperoxydes allyliques a longue chaine en presence de complexes de certains metaux de transition" ${ }^{n}$ - Chem. Phys. Lipids. 12 (1974) 239-248.

44. Hamberg, M.- "Vanadium-catalyzed transformation of 13(S)hydroperoxy-9(Z), 11 (E)-octadecadienoic acid: Structural studies on epoxy alcohols and trihydroxy acids".- Chem. Phys. Lipids 43 (1987) 55-67.

(Recibido: Diciembre 1990) 